\title{
Optical and Structural Characterization of Pin Photodetector Based on Germanium Nanocrystals for Third Generation Solar Cells
}

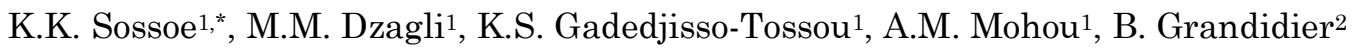 \\ ${ }^{1}$ Laboratoire de Physique des Composants à Semi-conducteurs (LPCS), Département de Physique, Faculté des \\ Sciences, Université de Lomé, BP 1515 Lomé, Togo \\ 2 Equipe Physique des Nanostructures et Composants Quantiques à l'Institut d'Electronique, de Microélectronique et \\ de Nanotechnologie (IEMN), IEMN CNRS, France
}

(Received 08 October 2016; published online 29 November 2016)

\begin{abstract}
We investigated the structural and optoelectronic properties of p-n germanium nanocrystals based junctions embedded between GaAs substrate and layers of $\mathrm{ZnO}: \mathrm{Al}$ or a-Si:H. Scanning electron microscopy and scanning tunneling microscopy were used on these junctions in this work. Calculations of tunneling current on the substrate showed effect of localized defects trapping Fermi level at the surface tending to make a semi-insulating substrate. The average value of the diameter of the Ge nanoparticle is around 12.5 $\mathrm{nm}$. These results lay the foundation for the development of solar cells which active part is made of GeNCs.
\end{abstract}

Keywords: Germanium nanocrystals, Photodetector, Renewable energy, Scanning tunneling spectroscopy, Solar cells.

DOI: $10.21272 /$ jnep.8(4(1)).04035

\section{INTRODUCTION}

Economic and ecological perspectives related to increasing energy costs and catastrophic climatic effects due to global warming are pushing us to find solutions to the issue of renewable energy. These solutions are becoming strategic for everyone with the socio-economic implications: (i) reduce the production of greenhouse gases, (ii) develop new energy solutions at a reasonable cost, (iii) have a sustainability of proposed solutions (iv) achieve energy independence [1].

For several years, many devices based on nanocrystals semiconductors have demonstrated the use of quantum dots in solar cells [2]. Nanoparticles have the potential to improve the efficiency of solar cells and photodetectors [1]. For example, studies on the introduction of quantum dots (QDs) based on III-V semiconductors in the pin structures showed that large energy absorption range may be obtained which increases the density of short-circuit current [3-10].

Thus, quantum dots offer a growing interest through the emergence of new concepts:

First, half of the intensity of sunlight is in the infrared region of the electromagnetic spectrum [11, 12]. Quantum dots can serve as intermediate electronic levels for the absorption of photons of energy less than the bandgap of the semiconductor in which they are embedded. By successively absorbing more photons, it is possible to generate high energy charge carriers to keep a high output voltage and therefore improve the output power of the cells [13].

Second, one can produce more excitons from a single photon. The formation of multiple excitons by absorbed photon requires that the photon energy is much larger than the band gap of the semiconductor. This phenomenon does not occur easily in semiconductors where excess energy is simply dissipated as heat before it can cause the formation of other electron-hole pairs. In the nanocrystals, the dissipated energy rate is significantly
PACS numbers: 61.72.uf, 68.37.Hk, 68.37.Ef, 71.15. - m, 73.63.Kv, 81.07.Bc reduced [14-16]. Thus, by using the quantum confinement properties, it is possible to generate a number of charge carriers from a single photon of high energy, when quantum dots constitute the active part of a solar cell [17]. Saeed et al. showed multiple generations of charge carriers through the use of quantum dots [18, 19].

Study of electronic properties of these nanocrystals are now crucial for achieving optimal generation and extraction of photo-excited charge carriers and then realize thin films of good quality. Germanium is an interesting material since it has a bandgap smaller than that of silicon and can form the quantum dots layer either by epitaxial growth techniques or by chemical synthesis. The interest of germanium nanocrystals (GeNCs) comes from the high mobility of charges carriers (electrons and holes), the best absorption of light due to its direct bandgap [20, 21] as well as compatibility with optoelectronics and photovoltaics applications.

In this study, we exhibit the optical and structural characterization of pin photodetector based on germanium nanocrystals in $n$-GaAs/GeNCs/a-Si:H and $p$-GaAs / GeNCs/ZnO:Al samples. These results show the potential of GeNCs in the manufacturing of 3G solar cells and show new types of absorber based on Ge.

\section{EXPERIMENTAL}

\subsection{Samples}

The prepared samples are $n$-GaAs/GeNCs/a-Si:H and the $p$-GaAs/GeNCs/n-ZnO:Al. These are precisely pin diodes whose substrate is GaAs layer and the intrinsic layer is made of GeNCs. Figure 1 shows the characteristic structure of the diode which are produced. The intrinsic layer of GeNCs is sandwiched between GaAs substrate and amorphous silicon or zinc oxide layers. The layer exposed to light is either amorphous silicon or zinc oxide.

\footnotetext{
*kodjo_sossoe@yahoo.fr
} 


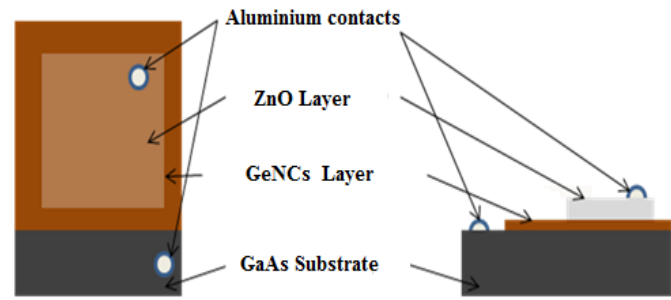

Fig. 1 - Structure of the sample $p$-GaAs/GeNCs/n-ZnO based on germanium nanocrystals

\subsection{Analysis Methods and Simulation Details}

Once the sample is prepared by deposition technics of thin layers and to access the structural and electrical properties, it is cleaved in ultrahigh vacuum. This allows performing measurements on the wafer. This cleavage technics is described in reference [22]. Scanning Electron Microscope (SEM) allowed imaging the cleaved surfaces of samples and reporting the nature of the layers. Analysis of these images allows access to the layer thickness and the size of the nanocrystals. Scanning Tunneling Microscopy (STM) is used to access more details of the sample than the SEM. Spectroscopic measurements were performed to extract surface states densities and the gap of semiconductor inspected. WsXM image processing software was used to process the images from Scanning Tunneling Microscopy Room Temperature (RT-STM). All these analysis were realized in physics laboratories at the Institute of Electronics Microelectronics and Nanotechnology (IEMN) and at the High Institute of Electronics of Digital (ISEN) at Lille in France.

Computations of the tunneling current were performed on p-doped GaAs substrate. These computations were conducted at Laboratory of Physics of Components and Semiconductor at University of Lomé (Togo), by improved code of SEMITIP Version 6 program of Professor FEENSTRA group [23, 24]. This permitted interpretations on the nature of localized defects observed on the substrate after the cleavage. This calculation involves the use of a finite element method assuming a metal tip serving as a hyperbolic shaped probe located at a typical distance of 0.7 to $0.8 \mathrm{~nm}$ from GaAs surface. The tunneling current was computed using an approach based on the formalism of Bardeen and Tersoff-Hamann approximation [25, 26]. Under these conditions the semiconductor was treated in an effective-mass approximation. Our simulation takes into account surface states associated with dangling bonds of $\mathrm{Ga}$ after cleavage. Due to relaxation of the cleaved surface these dangling bonds induce an energy continuum pushed beyond the conduction band. The surface states may be associated with localized defects are not taken into account by our simulation. Main input parameters used to perform the calculation of the tunneling current are the radius of curvature of the tip, the tip-sample distance, the contact potential, the doping level of semiconductor and density of surfaces states, respectively, $R, s, \Delta$, doping, surface state.
We present results from our samples through SEM and STM images and tunneling spectroscopy on $n$-GaAs substrate.

\subsection{SEM Analysis}

SEM images on the edge of $n-G a A s / G e N C s / a-S i: H$ sample (Figure 2 (a)) show n-doped GaAs substrate on which are deposited GeNCs. We noted that GaAs (110) cleaved surface is atomically flat. Therefore, upper structure is not impeding the cleavage. A first layer is visible and has a high graininess. It corresponds to the nanocrystal layer. It is covered with a compact layer with darker contrast: amorphous silicon.

SEM image of the cleaved sample $p$-GaAs/GeNCs/n$\mathrm{ZnO}: \mathrm{Al}$ has a roughness-free layer (GaAs substrate) on which are deposited GeNCs (Figure 2 (c)). ZnO layer which is a transparent structure cannot be seeing on this image although it is present (Figure 2 (c)).

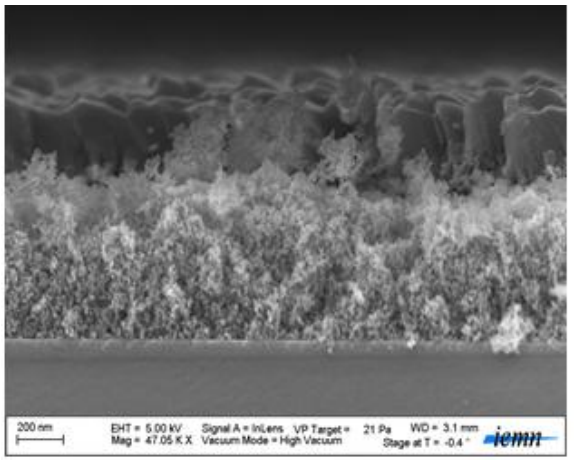

a

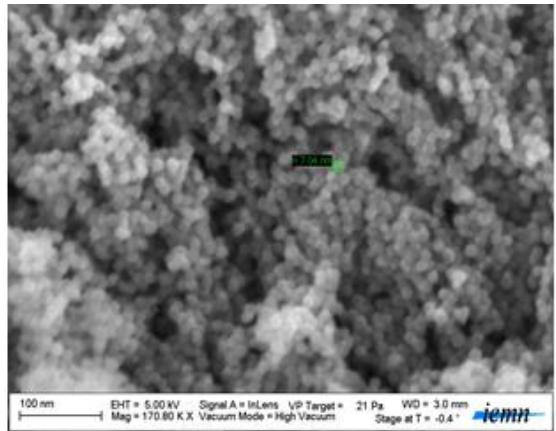

$\mathrm{b}$

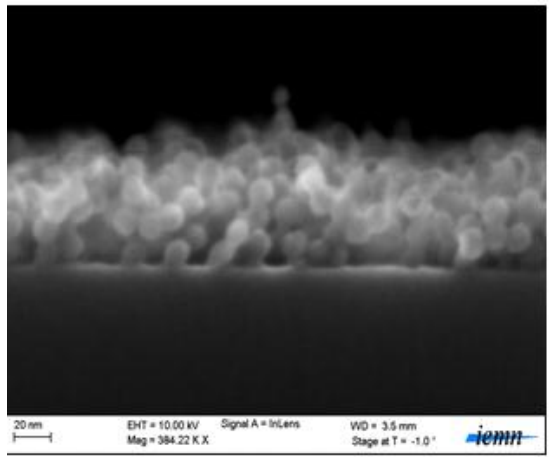

C

\section{RESULTS AND DISCUSSIONS}




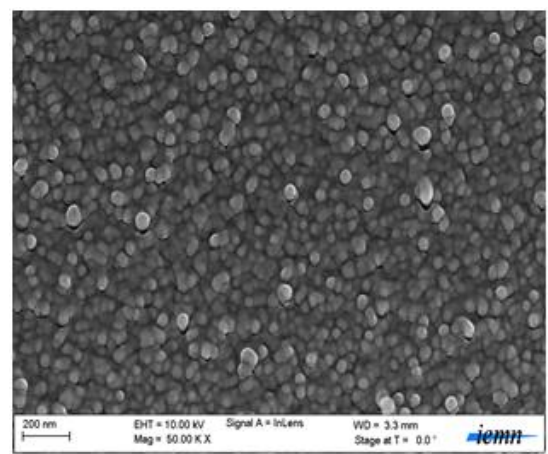

d

Fig. 2 - SEM images: (a)- $n$-GaAs/GeNCs/a-Si:H profile; (b) GeNCs from $n$-GaAs/GeNCs/a-Si:H; (c) $p$-GaAs/GeNCs $/ n$ ZnO:Al profile; (d) GeNCs from $p$-GaAs/GeNCs/ $n$-ZnO:Al

Top view shots on GeNCs layer of $n$-GaAs/GeNCs/a$\mathrm{Si}: \mathrm{H}$ and on $\mathrm{ZnO}$ layer of the $p$-GaAs/GeNCs/ $n-\mathrm{ZnO}: \mathrm{Al}$ are shown in Figure 2 (b) and Figure 2 (d), respectively. Analysis of these images provides an assessment of size and shape of Ge nanocrystals. These structures have spherical shapes with diameters between 7-15 nm, as confirmed by statistical analysis shown in Figure 3 . Overall, the average value of the diameter of the Ge nanoparticle is around $12.5 \mathrm{~nm}$. Also, one can see that the dispersion of nanocrystals is not very homogeneous because porosities can be observed on images.

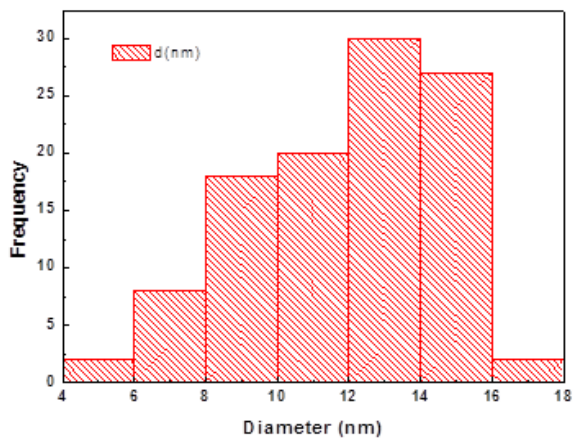

Fig. 3 - Statistics of nanocrystals size

\subsection{STM Analysis}

We have performed STM images on the $n$-GaAs/GeNCs/a-Si:H sample to appreciate details that can't be accessed by SEM. Scanning tunneling spectroscopy (STS) was used to interpret some observations on the substrate (Figure 4).

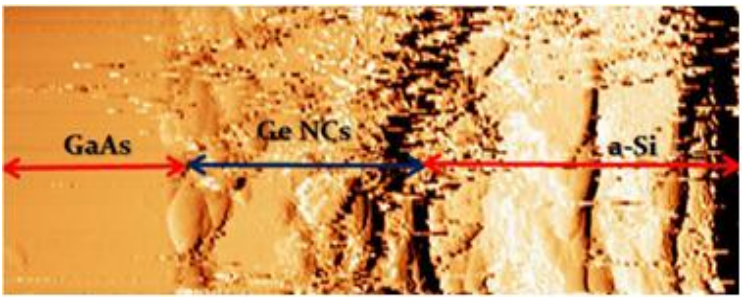

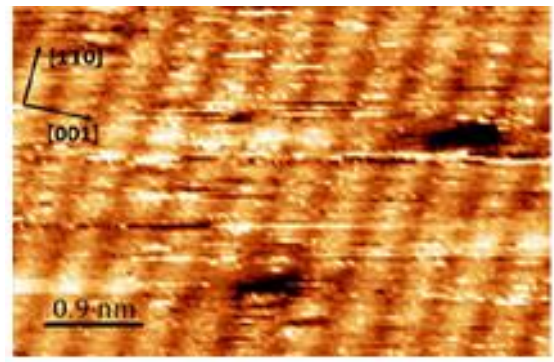

$\mathrm{b}$

Fig. 4 -RT-STM Images: (a) A large scale $n$-GaAs/GeNCs/a$\mathrm{Si}$ :H; (b) Atomic resolution on GaAs substrate (110). Tunneling conditions (a) $V_{T}=-2.5 \mathrm{~V}, I_{T}=1 \mathrm{pA}$; (b) $V_{T}=-2 \mathrm{~V}, I_{T}=4 \mathrm{pA}$

Figure 4 (a) is a zoom out that shows the three constituent layers of the sample analyzed. Due to the ease of cleavage of the GaAs in the atomic planes, its surface does not have asperities as observed on SEM image. Contrary, the intrinsic layer of nanocrystals which is not homogeneous reveals agglomerates. It is the same on the very rough amorphous silicon layer.

High resolution image, as shown on Figure 4 (b), was performed on the GaAs substrate highly n-doped shows further details on the substrate. It provides a vision of the surface topography. Point defects and the state of charge of these defects can be deduced from these STM images, according to various polarizations. It appears on our image obtained (Figure 4 (b)), atomic rows of $\mathrm{Ga}$ and As atoms in [1-10] crystallographic direction and surfaces defects like black spots. These black spots visible on the surface are located on atomic rows of arsenic and are associated with depressions. These are localized defects which interrupt the periodicity of the crystal lattice. This happens when one or more neighboring atoms of arsenic are absent from their usual incorporations sites. The defect is then associated with one or more vacancies.

\subsection{Tunneling Spectroscopy on $p$-GaAs sub- strate - tunneling current Simulation}

Scanning tunneling spectroscopy measurements were performed on highly p-doped GaAs layer. Experimental spectrum is shown in Figure 5 (red line). Two trends are observable: the current $(\mathrm{C})$ in the conduction band (CB) due to the transfer of electrons from the tip to the empty states of conduction band and current (V) in the valence band (VB) due to the passage of electrons from filled states of the VB to the tip. The steeper slope and the high amplitude of the current in the VB compared to that of CB indicate the high doping of the substrate. The Fermi level which is expected to be near the maximum of the valence band $(\mathrm{Ev})$ is slightly moved to the middle of the band gap at the surface. The fact that part of the voltage between the tip and the substrate is found in the substrate acts on the band bending. So, one can observe a shift of the Fermi level as noticed on our spectrum. 


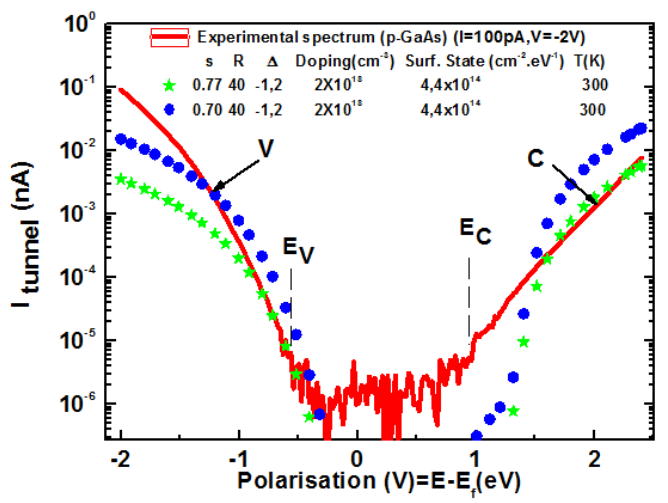

Fig. 5 - Comparison of experimental tunneling current spectrum on $\mathrm{p}$-GaAs layer (red line) and computed spectra (circle, star): the edges of VB and CB are indicated by Ev and Ec, the Fermi level is given by the position of $0 \mathrm{~V}$. Tunneling conditions (a) $V_{T}=-2 \mathrm{~V}, I_{T}=100 \mathrm{pA}$

In order to assist with the interpretation of the spectroscopic tunneling measurement, the tunneling current produced by tungsten tip near the GaAs (110) surface was computed with the Semitip code. The value attributed to the doping shows that the sample is highly doped. The density attributed to the dangling bonds is that used by Feenstra et al. [23, 24] on a cleaved surface of GaAs (110). Computation of I(V) characteristic of the tunneling current presents here two spectra according to the distance tip-surface (Figure 5 , circle, star). It is noticed that the amplitude of the tunneling current is not equal to that obtained experimentally according to the two distances tip-surface. However, in conduction band (positive bias), the current amplitude is greater than that obtained experimentally for a value of a distance equal to $0.70 \mathrm{~nm}$. In addition, the computed spectra show between $1 \mathrm{~V}$ and $1.5 \mathrm{~V}$ discrepancy compared to the experimental spectrum. It also notices that the crystalline quality of the substrate surface may be the cause of the discrepancy observed between theory and experience in valence band.

\subsection{Measure the Dark Current}

$\mathrm{I}(\mathrm{V})$ characteristic obtained in the dark on the pin structure of the $p$-GaAs/GeNCs/ZnO shows a threshold voltage around $0.3 \mathrm{~V}$ (see Figure 6). This threshold voltage value confirms that this is a germanium diode. At reverse bias, a leakage current occurs. This leakage related to minority carriers is created by thermal excitations. On one hand, this leakage arises from $-0.2 \mathrm{~V}$ and seems to slightly increase in intensity with polarization. This leakage would come from minority carriers' transport due to the electric field present in the depletion zone.

Thermally generated electrons in the $\mathrm{p}$ region reach the depletion region without undergoing recombination. They are driven by the electric field of the depletion region towards the $\mathrm{n}$ region. This gives rise to a negative current.

The same thing happens to holes generated thermally in the $\mathrm{n}$ region which will go along the $\mathrm{p}$ region. On the other hand the aluminum contact could also contribute to the presence of the leakage current observed in negative bias. The dark current is considered

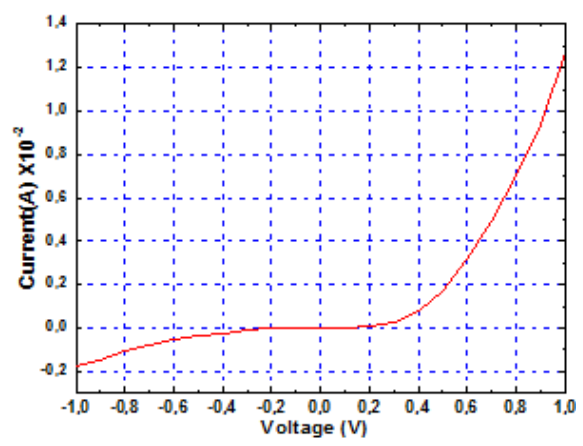

a

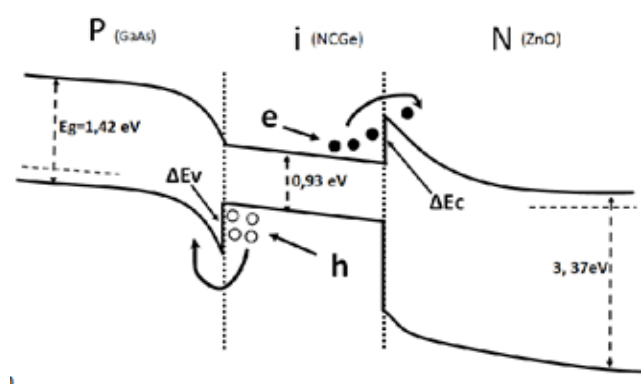

$\mathrm{b}$

Fig. 6 - Dark current in the $p$-GaAs/GeNCs/ZnO (a) and its associated band diagram (b)

as the sum of diffusion, generation-recombination and tunneling currents [27]. The band diagram of the pin structure is also shown in Figure 6.

\section{CONCLUSION}

In this present work, different techniques of optical and structural analysis on semiconductor surfaces are performed on two pin photodiodes, the $n$-GaAs/GeNCs/a$\mathrm{Si}: \mathrm{H}$ and the $p$-GaAs/GeNCs/n-ZnO:Al. A dark current measurement is performed on the $p$-GaAs/GeNCs $/ n$ $\mathrm{ZnO}: \mathrm{Al}$. These results announced interest in the use of germanium nanocrystals in the production of solar cells. Computations results of the tunneling current on the substrate have shown the effect of localized defects trapping the Fermi level at the surface tending to make the substrate semi-insulating. This affected the measured dark current.

\section{ACKNOWLEDGEMENTS}

This study was financially supported by the Agence Universitaire de la Francophonie (Grant No. BAO-201205-52220FT2E0). The authors thank Maxime Berthe, Jean-Philippe Nys, Yannick Lambert, Pierre Capiod, D. Stiévenard Etienne Quesnel for their technical input and fruitful discussions. We thank Prof. R. Feenstra for the use of his SEMITIP Program under the tunneling current simulations. 


\section{REFERENCES}

1. M.A. Green, Berlin: Springer-Verlag (2003)

2. A.J. Nozik, Nano Lett. 10, 2735 (2010).

3. M.A. Green, K. Emery, Y. Hishikawa, W. Warta, Prog. Photovoltaic. 17, 320 (2009).

4. M. Mazzer, K.W.J. Barnham, I.M. Ballard, A. Bessiere, A. Ioannides, D.C. Johnson, M.C. Lynch, T.N.D. Tibbits, J.S. Roberts, G. Hill, C. Calder, Thin Solid Films 511, 76 (2006).

5. D. Zhou, G. Sharma, S.F. Thomassen, T.W. Reenaas, B.O Fimland. Appl. Phys. Lett. 96, 061913 (2010).

6. A. Luque, A. Marti, Adv. Mater. 22, 160 (2010).

7. S.M. Hubbard, C.D. Cress, C.G. Bailey, R.P. Raffaelle, S.G. Bailey, D.M. Wilt, Appl. Phys. Lett. 92, 123512 (2008).

8. E. Antolin, A. Marti, C.R. Stanley, C.D. Farmer, E. Cánovas, N. López, P.G. Linares, A. Luque, Thin Solid Films 516, 6919 (2008)

9. M.Y. Levy, C. Honsberg, J. Appl. Phys. 104, 113103 (2008).

10. A. Martí, E. Antolín, C.R. Stanley, C.D. Farmer, N. López, P. Díaz, E. Cánovas, P.G. Linares, A. Luque, Phys. Rev. Lett. 97, 247701(2006).

11. A. Luque, A. Marti, Phys. Rev. Lett. 78, 5014 (1997)

12. A. Marti', L. Cuadra, A. Luque, Series in Optics and Optoelectronics, chapter 7 (Institute of Physics Publishing: Bristol: 2003).

13. A. Luque, A. Marti, Nat. Photonic. 5, 137 (2011).
14. Joseph M. Luther, Nano Lett. 7 No 6, 1779 (2007).

15. Minbiao Ji et al., Nano Lett. 9 No 3, 1217(2009).

16. R. Yu, Q. Lin, Siu-Fung Leung, Z. Fan, Nano Energ. 1, 57 (2012)

17. O. E. Semonin, et al., Science 334, 6062 (2011).

18. S. Saeed, C. Weerd, P. Stallinga, F. Spoor, A. Houtepen, L. Siebbeles, T. Gregorkiewicz, Light: Science \& Applications 4, e251 (2015).

19. M. Buljan, S. Pinto, A. Rolo, J. Martın-Sanchez, J. Grenzer, A. Mucklich, S. Bernstorff, Phys. Rev. B 82, 235407 (2010).

20. B. Zhang, S. Shrestha, G. Conibeer, Appl. Phys. Lett. 96, 261901 (2010).

21. W.L. Liu, P.F. Lee, J.Y. Dai, J. Wang, C.L. Choy, Z.T. Song, S.L. Feng, Appl. Phys. Lett. 86, 013110 (2005).

22. N.D. Jäger, PhD thesis, Forschungszentrum Jülich in der Helmholz-Gemeinschaft (2003).

23. R.M. Feenstra, http://www.andrew.cmu.edu/user/feenstra/semitip_v6/UniI ntSC2/

24. R.M. Feenstra, Phys. Rev. B 50, 4561 (1994)

25. J. Tersoff, D.R. Hamann, Phys. Rev. Lett. 50, 1998 (1983).

26. J. Tersoff, D.R. Hamann, Phys. Rev. B 31, 805 (1985)

27. M.M. Dzagli, M.A. Mohou, J. Rech. Sci. Univ. Lomé (Togo), Série E 12 (1), 17 (2010). 